\title{
Recherche de la xanthine oxydase appliquée à la mise en évidence du lait de vache ajouté au lait de chèvre
}

\author{
Mise au point d'un microtest
}

\author{
par \\ D. MONGET $\left({ }^{*}\right)\left({ }^{* *}\right)$, M. GELIN $\left({ }^{* * *}\right)$ et P. LAVIOLETTE $(*)$
}

\section{INTRODUCTION}

Plusieurs méthodes pour déceler des mélanges de laits de chèvre et de vache ont déjà été mises au point à la demande du Ministère de l'Agriculture et des Coopératives Laitières. La technique officielle de Pierre et Portmann (1970) utilise l'électrophorèse sur gel de polyacrylamide qui permet de préciser l'origine du lait en fonction des propriétés migratoires de certaines protéines qu'il contient. Cette technique a été récemment complétée par une méthode sérologique, le CV Test, ou Chèvre-Vache Test (Levieux, 1977) dont le principe est celui de la réaction d'inhibition de l'hémagglutination, en présence d'un antisérum anti-lait de vache et de globules rouges de Poule sensibilisés par des immunoglobulines bovines. Le même auteur a également proposé l'immunodiffusion radiale en milieu gélosé contenant un antisérum spécifique du lait de vache. Avec ces techniques immunologiques, pratiquées sur le lactosérum, il est possible de détecter 1 p. 100 , voire 1 p. 1000 , de lait de vache ajouté à du lait de chèvre.

Le test que nous proposons repose sur un principe très différent de celui des méthodes mentionnées ci-dessus. Il est basé sur la mise en évidence colorimétrique d'une enzyme, la xanthine oxydase, dont la concentration toujours plus élevée dans le lait de vache que dans le lait de chèvre, permet de distinguer les deux types de laits.

(*) Laboratoire de Biologie - 406, Institut National des Sciences Appliquées 69621 Villeurbanne cedex.

(**) Nouvelle adresse : Laboratoire de Recherche API, La Balme-les-Grottes 38390 Montalieu-Vercieu.

${ }^{(* * *)}$ Conseiller Scientifique de Scoff - U.R.C.V.L. - Graqlait. 


\section{MATERIEL ET METHODES}

La détection de la xanthine oxydase fait partie d'une série de tests " déshydrogénases " récemment mis au point (Monget, 1978), utilisant le matériel API ZYM (*) (Monget, 1975). L'oxydation de la xanthine en acide urique en présence de nitroblue tétrazolium (NBT) entraîne une réduction irréversible de ce dernier en formazan coloré en violet.

Le test se présente sous forme d'une galerie de dix cupules, contenant chacune la xanthine sous forme déshydratée, à la concentration de $2 \mathrm{mM}$ dans un tampon phosphate $0,1 \mathrm{M}, \mathrm{pH} \mathrm{7,5}$. Le matériel est conservé à $+4^{\circ} \mathrm{C}$ jusqu'au moment de l'utilisation.

\section{a) Pratique du test appliqué au lait}

L'échantillon de lait de chèvre est emprésuré $(1 \mathrm{ml}$ de présure concentrée pour 101 de lait) et mis au bain-marie à $38^{\circ} \mathrm{C}-40^{\circ} \mathrm{C}$ pendant quelques minutes. $100 \mu \mathrm{l}$ de lactosérum sont introduits dans une cupule prête à l'emploi. On ajoute alors une goutte du réactif de révélation ayant pour composition :

- $60 \mathrm{mg}$ nitroblue tétrazolium (SIGMA) ;

- $3 \mathrm{mg}$ phénazine méthosulfate (SIGMA) ;

- $100 \mathrm{ml} \mathrm{H}_{2} \mathrm{O}$.

La cupule est ensuite placée dans une étuve à $37^{\circ} \mathrm{C}$. Après une incubation de $15 \mathrm{mn}$, le résultat du test est apprécié par simple lecture à l'œil. Une réaction positive, qui se traduit par l'apparition d'une coloration violette, indique la présence de lait de vache dans l'échantillon. Cette coloration est d'autant plus intense que la quantité de lait de vache ajoutée est importante. L'utilisation de témoins, constitués de mélanges de laits de titre connu et analysés en même temps que l'échantillon à tester permet une lecture semi-quantitative. Ces témoins biologiques peuvent d'ailleurs être avantageusement remplacés par une solution d'ascorbate de sodium à différentes concentrations dans un tampon TRIS - $\mathrm{HCl} \mathrm{0,1} \mathrm{M,} \mathrm{pH} \mathrm{8,5.} \mathrm{L'ascorbate} \mathrm{réduit}$ spontanément le nitroblue tétrazolium en formazan (cf. tab. 1).

\section{b) Pratique du test appliqué aux fromages}

Le test peut être également appliqué aux fromages frais. Dans ce cas, le protocole expérimental est le suivant : $1 \mathrm{~g}$ de fromage est homogénéisé dans un tube de Potter avec $10 \mathrm{ml} \mathrm{H}_{2} \mathrm{O} .40 \mu \mathrm{l}$ de la sus-

(*) Le dispositif API ZYM est commercialisé par API System, La Balme-lesGrottes - 38390 Montalieu-Vercieu (France). 
pension obtenue et une goutte de réactif de révélation sont introduits dans la cupule utilisée pour le test. L'incubation est effectuée à $37^{\circ} \mathrm{C}$ pendant $1 \mathrm{~h}$.

\section{Remarques :}

- en fin d'incubation de l'échantillon, il est possible de bloquer la réaction enzymatique en ajoutant dans la cupule une goutte d' $\mathrm{HCl}$ $1 \mathrm{~N}$. La coloration reste alors stable dans le temps ;

- le mélange de nitroblue tétrazolium et de phénazine méthosulfate est très sensible à la lumière et doit de ce fait être conservé à l'obscurité totale. Stocké à $+4^{\circ} \mathrm{C}$, il peut être utilisé pendant 6 mois .

\section{RESULTATS}

\section{a) Contrôle de mélanges de laits de titre connu}

Nous avons recherché la xanthine oxydase dans différents mélanges de laits de chèvre et de vache. Les résultats sont consignés au tableau 1, dans lequel nous avons également fait figurer la quantité d'ascorbate de sodium nécessaire pour obtenir une production de formazan à partir du nitroblue tétrazolium identique à celle observée avec chacun des mélanges de laits. Les réponses au test sont notées de 0 à +++++ , selon l'intensité de la coloration, après une incubation de $15 \mathrm{mn}$ à $37^{\circ} \mathrm{C}( \pm=$ trace, $0< \pm<+)$.

\section{TABLEAU 1}

Etalonnage du test à la xanthine oxydase

\begin{tabular}{c|c|c}
\hline $\begin{array}{c}\text { Pourcentage } \\
\text { lait de vache/lait de chèvre }\end{array}$ & Note & $\begin{array}{c}\text { Quantité d'ascorbate } \\
\text { de sodium par cupule } \\
(\mathrm{en} \mu \mathrm{g} / 100 \mu \mathrm{l})\end{array}$ \\
\cline { 2 - 2 } 0 & $0 \grave{\mathrm{à}}+$ & 0 \\
1 & \pm & 2,0 \\
2 & + & 4,0 \\
5 & $+\grave{a}++$ & 6,5 \\
10 & ++ & 8,5 \\
20 & +++ & 12,5 \\
100 & +++++ & 25 \\
& & \\
\hline
\end{tabular}


Ce tableau montre qu'il est parfaitement possible de détecter la présence de lait de vache dans du lait de chèvre, en raison de l'importante différence observée entre les taux de xanthine oxydase chez ces deux espèces.

Le seuil de sensibilité de la méthode est atteint pour un mélange ayant un titre de 2 p. 100.

\section{b) Etude de la reproductibilité du test sur des laits de chèvre}

Le test à la xanthine oxydase a été pratiqué à intervalles réguliers pendant une année complète sur quarante chèvres de race alpine, appartenant à trois producteurs différents. Les laits utilisés sont garantis sans addition de lait de vache.

En dehors de la période de gestation et des premiers jours qui suivent la mise-bas, tous les échantillons se sont révélés négatifs. Il faut entendre par "négatif " un test dont le résultat se traduit, soit par l'absence totale de coloration, soit par la mise en évidence d'une quantité de formazan correspondant à une valeur inférieure au seuil de 2 p. 100.

A la fin de la période de lactation et au début de la reprise de celle-ci, le résultat du test chez 20 p. 100 des animaux atteint des valeurs équivalentes à celles observées pour des mélanges de laits vache/chèvre compris entre 2 p. 100 et 5 p. 100 .

\section{c) Contrôle des fromages de chèvre}

Nous avons demandé à un producteur de nous fabriquer des fromages à partir de mélanges de laits contenant respectivement 0 p. 100,5 p. 100,10 p. 100 et 20 p. 100 de lait de vache. Les analyses ont été effectuées, soit sur des fromages frais de $4 \mathrm{j}$, soit sur des fromages secs de 1 mois.

Les résultats du tableau 2 indiquent que le test de la xanthine oxydase peut être utilisé sur des fromages frais pour un contrôle semi-quantitatif de la teneur en lait de vache. Par contre, il ne s'applique pas aux fromages secs dont les protéines, et donc les enzymes, sont dégradées au cours de l'affinage.

\section{d) Influence de la pasteurisation}

En cas de mélange, les résultats précédents n'ont de valeur que si du lait cru de vache a été ajouté au lait de chèvre. Mais il est également important de connaître la réponse du test en présence de lait de vache pasteurisé. Pour ce faire, nous avons chauffé du lait cru entre $75^{\circ} \mathrm{C}$ et $81^{\circ} \mathrm{C}$ au bain-marie pendant $20 \mathrm{~s}$ dans un tube capillaire en verre très fin (diamètre intérieur $2 \mathrm{~mm}$, épaisseur de la paroi $0,2 \mathrm{~mm}$ ), à défaut d'avoir pu disposer d'un pasteurisateur expérimental. Le lactosérum est ensuite soumis au test de la xanthine oxydase. L'étude de la thermo-sensibilité de l'enzyme figure au tableau 3. 


\section{$T A B L E A U 2$}

Analyse de fromages obtenus à partir de différents mélanges de laits vache/chèvre

\begin{tabular}{c|c|c}
\hline $\begin{array}{c}\text { Pourcentage de lait } \\
\text { de vache entrant dans la } \\
\text { composition du fromage } \\
\text { de chèvre }\end{array}$ & $\begin{array}{c}\text { Fromage frais }\left(^{*}\right) \\
(4 \mathrm{j})\end{array}$ & $\begin{array}{c}\text { Fromage sec }\left(^{*}\right) \\
(1 \text { mois })\end{array}$ \\
\hline 0 & 0 & 0 \\
5 & + & \pm \\
10 & $\begin{array}{c}\text { à }++ \\
++\grave{a}+++\end{array}$ \\
\hline
\end{tabular}

(*) Note de 0 à +++++ , selon l'intensité de la coloration, après une incubation de $1 \mathrm{~h}$ à $37^{\circ} \mathrm{C}$. $\pm=$ trace $0< \pm<+$ ).

TABLEAU 3

Action de la température de pasteurisation sur l'activité de la xanthine oxydase du lait de vache

Température de pasteurisation du lait de vache
Test de la xanthine oxydase $(*)$

$81^{\circ} \mathrm{C}$
$80^{\circ} \mathrm{C}$
$79^{\circ} \mathrm{C}$
$78^{\circ} \mathrm{C}$
$77^{\circ} \mathrm{C}$
$76^{\circ} \mathrm{C}$
$75^{\circ} \mathrm{C}$

$$
\begin{gathered}
0 \\
0 \\
+ \\
++ \\
+++ \\
++++ \\
+++++
\end{gathered}
$$

(*) Note de 0 à +++++ , selon l'intensité de la coloration, après une incubation de $15 \mathrm{mn}$ à $37^{\circ} \mathrm{C}$. 
Si la xanthine oxydase est stable en-dessous de $76^{\circ} \mathrm{C}$, elle est par contre totalement inactivée lorsque le lait est chauffé à une température égale ou supérieure à $80^{\circ} \mathrm{C}$ pendant $20 \mathrm{~s}$. Ainsi la pasteurisation haute, qui est la plus généralement effectuée dans les laiteries (quelques secondes à plus de $80^{\circ} \mathrm{C}$ ), entraîne la dénaturation de l'enzyme. Le test ne peut donc être appliqué lorsque du lait de vache pasteurisé a été ajouté à du lait de chèvre.

\section{DISCUSSION ET CONCLUSION}

Ce travail a été précédé d'une étude comparative non publiée de l'équipement enzymatique des laits de vache et de chèvre, établi à partir des soixante-dix caractères API ZYM que nous avons mis au point (Monget, 1978). Parmi cette gamme très large de tests, nous avons sélectionné l'un d'entre eux, particulièrement intéressant pour la détection des mélanges de laits de ces deux espèces. La xanthine oxydase est une enzyme dont le taux dans le lait de vache est beaucoup plus élevé que celui trouvé dans le lait de chèvre. Ses propriétés ont surtout été étudiées dans le lait de vache (Pien, 1945 ; Zittle et al., 1956) mais l'existence de cette différence de concentration de l'enzyme entre les laits de chèvre et de vache, bien que connue (Owen et Proudfoot, 1968) n'a jamais été mise à profit pour contrôler les fournitures de lait de chèvre.

Le test enzymatique proposé vient compléter les techniques actuelles d'électrophorèse de Pierre et Portmann (1970) et sérologique de Levieux (1977). La méthode s'applique aux laits crus de vache et de chèvre et n'est adaptée aux laits pasteurisés que si ces derniers ont subi une pasteurisation basse (moins de $76^{\circ} \mathrm{C}, 20 \mathrm{~s}$ ). Ce résultat confirme l'étude de la thermo-sensibilité de la xanthine oxydase, effectuée par Pien (1945), qui a jadis proposé la recherche de l'enzyme, également appelée enzyme de Schardinger, comme méthode de contrôle de la pasteurisation du lait. De nos jours, on préfère utiliser le test de la peroxydase par la méthode de Dupouy (voir Pien, 1945) plus facile et rapide à mettre en œuvre, et mieux adapté au contrôle de la pasteurisation haute.

Sans atteindre la sensibilité de 1 p.1 000 du test de Levieux (1977), l'épreuve à la xanthine oxydase permet de détecter deux parties de lait de vache ajoutées à cent parties de lait de chèvre. Cette sensibilité, comparable à celle de la méthode de Pierre et Portmann, est suffisante pour déceler des fraudes économiquement rentables.

L'interprétation du test est difficile au cours de la période de gestation et durant les premiers jours qui suivent la mise-bas. Dans certains cas, il conduit à des résultats faussement positifs, équivalents à la mise en évidence de mélange de laits vache/chèvre de l'ordre de 5 p. 100 . Cet inconvénient est cependant mineur puisque les animaux ne sont pas soumis à la traite au cours de cette période. 
Comme dans les autres méthodes, le test enzymatique est effectué sur le lactosérum. Le lait de chèvre renferme en effet une faible quantité de xanthine oxydase, ainsi que des composés réducteurs non enzymatiques, dont la présence se traduit par la production de formazan à partir du nitroblue tétrazolium. Ce fond parasite est éliminé en précipitant la caséine, qui entraîne avec elle la plupart des éléments indésirables au bon fonctionnement du test.

L'analyse enzymatique peut être pratiquée sur des fromages frais avec un seuil de sensibilité voisin de 5 p. 100. Lorsque les fromages sont à un stade de maturation avancé et ont commencé à subir un début de protéolyse, la xanthine oxydase est dénaturée et le test n'a plus de signification.

En dépit de ses limites, le test de la xanthine oxydase possède de nombreux atouts. L'utilisateur pourra en particulier apprécier sa simplicité, sa rapidité et son faible coût, chaque fois qu'il aura à effectuer un contrôle de lait de chèvre avant transformation.

Aux côtés des techniques habituellement utilisées, les tests enzymatiques, dérivés de la méthode API ZYM, devraient apporter un intéressant outil de complément à l'Industrie Laitière, alors que les contrôles systématiques deviennent de plus en plus nombreux. C'est ainsi que tout récemment nous avons mis au point deux microtests " phosphatase alcaline " et " peroxydase » destinés à la surveillance de la pasteurisation du lait de vache (Monget et Laviolette, 1978). De plus, les résultats d'une étude préliminaire nous autorisent à penser que l'épreuve à la xanthine oxydase doit également pouvoir s'appliquer à la détection d'une éventuelle addition de lait de vache dans du lait de brebis.

\section{Rés u m é}

Un microtest "xanthine oxydase ", simple et rapide, utilisant le nitroblue tétrazolium, est proposé pour mettre en évidence la présence de lait de vache éventuellement ajouté à du lait de chèvre. Il s'applique au lait cru et pasteurisé (maximum : $75^{\circ} \mathrm{C}, 20 \mathrm{~s}$ ), ainsi qu'aux fromages frais, et permet de déceler deux parties de lait de vache dans cent parties de lait de chèvre.

\section{S u m m a ry}

THE SEARCH FOR XANTHINE OXIDASE, APPLIED TO THE DEMONSTRATION OF THE PRESENCE OF COWS'MILK ADDED TO GOATS'MILK REALISATION OF A MICROTEST

A «xanthine oxidase» microtest, simple and rapid, using nitroblue tetrazolium, is proposed to show the presence of cows'milk possibly added to goats'milk. It applies to raw and to pasteurized milk ( $75^{\circ} \mathrm{C}$, 
$20 \mathrm{~s}$, at the most), as well as to fresh cheeses and permits the detection of two parts of cows' milk in one hundred parts of goats'milk.

Reçu pour publication en décembre 1978.

\section{Bibliographie}

Levieux (D.) (1977). - Une nouvelle technique de détection de l'adultération des laits de chèvre et de brebis. Dossiers de l'Elevage, 2, 37-46.

Monget (D.) (1975). - Différence d'activités enzymatiques entre deux lignées cellulaires d'insectes : Antheraea eucalypti et Malacosoma disstria, (Lepidoptera). C.R. Acad. Sci., 281, 651-654, série D.

Monget (D.) (1978. - Mise au point d'une microméthode de détection et de mesure d'activités enzymatiques (API ZYM). Résultats obtenus dans différents domaines d'application. Thèse de Docteur-Ingénieur, Université de Lyon, $152 \mathrm{p}$.

Monget (D.) et Laviolette (P.) (1978). - Mise au point de microtests " phosphatase alcaline " et " peroxydase " pour le contrôle de la pasteurisation du lait de vache. Le Lait, 58, 595-605.

OWen (E. C.) and Proudfoot (R.) (1968). - Anaerobic procedure for estimating xanthine oxidase in milk using triphenyltetrazolium chloride (TTC). Brit. J. Nutr., 22, 331-332.

PIEN (J.) (1945). - Le contrôle de la pasteurisation du lait et de la crème. Le Lait, 25, 311-320.

Pierre (A.) et Portmann (A.) (1970). - Emploi de l'électrophorèse en gel de polyacrylamide pour mettre en évidence et doser le lait de vache ajouté au lait de chèvre. Application au cas des fromages. Annls Technol. Agric., 19, 107-130.

Zittle (J. H.), Dellamonica (E. S.), Custer (J. H.) and Rudd (R. K.) (1956). Determination of xanthine oxidase in milk with triphenyltetrazolium chloride. J. Dairy Sci., 39, 522-527. 